Dear Editor,

\title{
Axiomatic principles for a market model
}

\section{Introduction}

Despite many attempts, the consistent and global modelling of markets remains an open problem. In particular it remains a challenge to find a simple, probabilistic approach to general market modelling which captures the essence of reality. The literature on financial market modelling is quite extensive. To mention a few references one could for instance refer to the work by Sharpe (1964), Lintner (1965), Merton (1973), Black and Scholes (1973), and Ross (1976).

This letter indicates an approach to market modelling that allows for the inclusion of typical market features in a consistent framework. To illustrate the approach we introduce a simple goods market model that allows us to discuss key relationships between stochastic volatility, trading rate and forward rates that are consistent with the six axiomatic principles we are going to formulate. These principles characterize fundamental properties a market should have. They cannot be proven but they are consistent with empirical evidence and conform to common sense. Their consequences will be fully explored elsewhere in the context of different markets.

\section{A market model}

For illustration purposes let us consider a simple example of a goods market model. Suppose that there are $N$ goods to be traded in the market. We denote by $S_{t}^{j, i}$ the price at time $t \geq 0$ for one unit of the $j$ th good expressed in units of the $i$ th good and assume that

$$
S_{t}^{j, i}=S_{t}^{j, k} S_{t}^{k, i}
$$

for all $i, j, k \in\{1,2, \ldots, N\}$, where $S_{t}^{i, i} \equiv 1$ and $S_{0}^{j, i}>0$ is a given value. The price $S_{t}^{j, i}$ can be interpreted as the number of units of the $i$ th good which can be exchanged for one unit of the $j$ th good. Thus in this market we treat each of the goods as a type of currency in its own right.

Furthermore, we introduce the trading rate $Y_{t}^{i}$ of the $i$ th good at time $t$, that is the number of units of the $i$ th good traded per unit time at time $t \geq 0, i \in\{1,2, \ldots, N\}$, where $Y_{0}^{i}>0$ is given.

We call the product of trading rate and the price of a good its trading value. Let us assume that the relative trading value $q_{i}$ of the $i$ th good is a constant and defined by the ratio

$$
q_{i}=\frac{Y_{t}^{i} S_{t}^{i, j}}{\sum_{k=1}^{N} Y_{t}^{k} S_{t}^{k, j}}>0,
$$

for all $i, j \in\{1,2, \ldots, N\}$ and $t \geq 0$. Note that the relative trading value $q_{i}$ is independent of

Received 16 April 1997; revision received 11 February 1998. 
the choice of $j$. Then, it is straightforward to show that the price $S_{t}^{j, i}$ has the form

$$
S_{t}^{j, i}=\frac{q_{j}}{Y_{t}^{j}} \frac{Y_{t}^{i}}{q_{i}},
$$

for all $i, j \in\{1,2, \ldots, N\}$ and $t \geq 0$.

We also assume that each good has a given constant average trading rate $X^{i}$, $i \in\{1,2, \ldots, N\}$. The standardized trading rate $Z_{t}^{i}$ of the $i$ th good at time $t$ shall then be defined as

$$
Z_{t}^{i}=\frac{Y_{t}^{i}}{X^{i}}
$$

and it is assumed to follow a linear mean-reverting stochastic differential equation of the form

$$
\mathrm{d} Z_{t}^{i}=v^{2}\left(1-Z_{t}^{i}\right) \mathrm{d} t+Z_{t}^{i} \sigma_{t}^{i} \mathrm{~d} W_{t}^{i},
$$

for all $i \in\{1,2, \ldots, N\}, t \geq 0$. Here the processes $W^{1}, W^{2}, \ldots, W^{N}$ denote independent standard Wiener processes on a filtered probability space $\left(\Omega, \mathcal{F}, \underline{\mathcal{F}}=\left(\mathcal{F}_{t}\right)_{t \geq 0}, P\right)$, fulfilling the usual conditions. The parameter $v>0$ is taken to be constant.

The volatility $\sigma_{t}^{i}$ of the $i$ th good at time $t \geq 0$ shall be given by the expression

$$
\sigma_{t}^{i}=\frac{v}{\sqrt{Z_{t}^{i}}},
$$

for all $i \in\{1,2, \ldots, N\}$ and $t \geq 0$. This choice for the volatility will be discussed further in Section 3.

As previously mentioned we treat each of the goods as a type of currency. In the same way that a forward rate based on a lending rate can be defined for a particular currency we can similarly define forward rates with respect to each of the goods traded in our market. For the $i$ th good the continuously compounding forward rate $f_{t}^{i}$ at time $t$ is chosen to have the form

$$
f_{t}^{i}=\left(\sigma_{t}^{i}\right)^{2}
$$

for all $i \in\{1,2, \ldots, N\}$ and $t \geq 0$.

As with relation (2.6), equation (2.7) will be further explained in what follows.

The dynamics of this simple goods market model can be derived on the basis of six axiomatic principles that we will describe in the remaining part of this letter.

\section{Principles}

First we introduce a principle of inertia that characterizes the dynamics of the standardized trading rate of an asset, in our case a good.

First Principle. The standardized trading rate of an asset maintains a mean-reverting dynamics that remains stationary as long as its volatility is stationary.

This principle characterizes the long term undisturbed evolution of the trading rate. Note that the linear mean-reverting stochastic differential equation (2.5) is consistent with this principle. 
To specify the global interaction in the market one needs a second principle. Let us fix $i, j \in\{1,2, \ldots, N\}$ and $t \geq 0$ for the moment. Then $B_{t}^{i}$ represents the savings account of the $i$ th good at time $t$ that is defined by the equation

$$
\mathrm{d} B_{t}^{i}=B_{t}^{i} f_{t}^{i} \mathrm{~d} t
$$

with $B_{0}^{i}=1$. We denote by $\bar{S}_{t}^{j, i}$ the discounted price given by

$$
\bar{S}_{t}^{j, i}=\frac{S_{t}^{j, i}}{B_{t}^{i}}
$$

The increments of the discounted cumulative cost $\bar{C}_{t}^{j, i}$, in our case $\bar{C}_{t}^{j . i}=-\bar{S}_{t}^{j, i}$, can be positive or negative due to the stochasticity of the price $S_{t}^{j, i}$. Now we can form the relative cost increment,

$$
R_{t, \Delta}^{j, i}=-\left(\frac{\bar{S}_{t+\Delta}^{j, i}-\bar{S}_{t}^{j, i}}{\bar{S}_{t}^{j, i}}\right)
$$

over the small time interval $(t, t+\Delta]$ of length $\Delta>0$. We note that $R_{t, \Delta}^{j, i}$ denotes, in our example, the negative return of the discounted price of the $j$ th good if measured in units of the $i$ th good.

If the discounted cumulative costs were constant, then we would only have to pay the purchasing price for a good at the beginning; no further costs would arise over time. However the discounted cumulative costs are not constant because the prices of goods are stochastic. Instead of assuming constant discounted cumulative costs in a market it is more realistic to assume that discounted cumulative costs are kept as close as possible to their initial value. Such a market could be called fair. To formalize this assumption in mathematical terms we will minimize the squared relative cost increments. More precisely we introduce the (value weighted) average squared relative cost increment $Q_{t, \Delta}$ at time $t \geq 0$, that is given in the form

$$
Q_{t, \Delta}=\frac{1}{\Delta} \sum_{i=1}^{N} \frac{q_{i}^{2}}{X^{i}} \sum_{k=1}^{N} Y_{t}^{k} S_{t}^{k, i}\left(R_{t, \Delta}^{k, i}\right)^{2} .
$$

Since the average squared relative cost increment is random we introduce the market risk $L_{t, \Delta}$ as its expectation

$$
L_{t, \Delta}=E\left(Q_{t, \Delta}\right)
$$

for $t \geq 0$ and $\Delta>0$. Then we assume that the market has the global objective to minimize the market risk, that is

$$
L_{t, \Delta}^{*}=\operatorname{ess} \inf L_{t, \Delta}
$$

for $t \geq 0$ and $\Delta>0$, where we used the essential infimum. Finally we define the minimal market risk $\Gamma_{t}$ at time $t \geq 0$ as

$$
\Gamma_{t}=\lim _{\Delta \rightarrow 0} L_{t, \Delta}^{*}
$$


This is related to the concept of local risk minimization that was introduced for derivatives by Föllmer and Sondermann (1986) and Föllmer and Schweizer (1991).

It is apparent that participants in a market have their goals (e.g. to maximize the growth rate of their wealth) and act competitively according to their strategies. In the presence of large traders or groups of traders with similar objectives it is therefore appropriate to model the market dynamics as a stochastic game. The objectives of individual market participants, when aggregated, lead to a global market objective which is to minimize some resulting payoff function that we shall call market risk. Taking this into account we then formulate the following market risk minimization principle.

Second Principle. The dynamics of a market represents a stochastic game that is characterized by the individual goals of the participants and the global objective to minimize the market risk.

Interpreting the volatility as a control parameter one can show that the choice for the volatility given in (2.6) minimizes the market risk in our example. With this choice the value weighted squared volatility $v_{t}^{i}$ is of the form,

$$
v_{t}^{i}=q_{i} \frac{Y_{t}^{i}}{X^{i}}\left(\sigma_{t}^{i}\right)^{2}=v^{2} q_{i}
$$

for all $t \geq 0$ and $i \in\{1,2, \ldots, N\}$. Note that the value weighted volatility is in an equilibrium state; in fact in our example it is constant. Our minimization procedure is therefore related to the well-known maximum entropy principle.

Furthermore, one can show that the minimization of the market risk with respect to the forward rates yields (2.7). That is, the forward rate for the $i$ th good is equal to the squared volatility $\left(\sigma_{t}^{i}\right)^{2}$ of the standardized trading rate $Z_{t}^{i}$. This coincides with a result in Platen and Rebolledo (1996) based on the arbitrage information minimization principle. This principle minimizes the Kullback-Leibler distance between some martingale probability measure and the given probability measure $P$. In our example one can show that this difference vanishes completely and the measure $P$ becomes the martingale measure for the discounted price $\bar{S}_{t}^{j, i}$. That is, we have

$$
E\left(\bar{S}_{t}^{j, i} \mid \widetilde{F}_{s}\right)=\bar{S}_{s}^{j, i}
$$

for all $j, i \in\{1,2, \ldots, N\}$ and $t \geq s \geq 0$. In this sense we have recovered a version of the well-known no-arbitrage pricing principle (see Ross (1976)) from our market risk minimization procedure. We remark that the discounted cumulative costs also represent martingales under the given probability measure $P$.

We can reasonably assume that the various goods are exerting market forces on each other. These forces lead to corresponding movements in their trading values (i.e. the product of trading rate and price). If two goods interact, that is they exert market forces on each other, then the corresponding trading values are essential to explain the impact of this interaction on the market dynamics. For instance if the market is losing interest in a specific good but is instead developing demand for another good with a much smaller trading value, then the expected rate of return of the trading value of the latter will be severely influenced by this shift in demand, whereas the expected rate of return of the trading value of the former good might only show a small change.

The following market force principle clarifies the general impact of market forces on the dynamics of the trading value of an asset. 
Third Principle. The expected rate of return of the trading value of an asset is directly proportional to the market force acting on the asset, is inversely proportional to its trading value, and has the same direction as the market force.

For instance in our goods market the market force $F_{t}^{j, i}$ that acts on the $j$ th good at time $t$, if expressed with respect to the $i$ th good, is equal to the product of the trading value of the $j$ th good and its expected rate of return. That is by (2.3)-(2.5) we have in our example

$$
F_{t}^{j, i}=\left(Y_{t}^{j} S_{t}^{j, i}\right) v^{2}\left(\left(Z_{t}^{i}\right)^{-1}-1\right)=\frac{q_{j}}{q_{i}} v^{2}\left(X^{i}-Y_{t}^{i}\right)
$$

for all $i, j \in\{1,2, \ldots, N\}$ and $t \geq 0$.

If we call the market force that is exerted on an asset or good by a second asset the action and the market force exerted on the second asset by the first asset the reaction, then we can state the principle of action and reaction.

Fourth Principle. To every action there is an equal and opposite reaction.

It can be argued that the value of goods (and also the monetary value) can be viewed as representing energy since they can be exchanged for work. We can measure risk that is related to a specific uncertainty by the negative price for a corresponding derivative that offsets this uncertainty. In our goods market it could be the risk that the price of a certain good falls below some critical value at some future time. This risk can be offset by a corresponding European put option. With this formulation, risk can be expressed as the value of goods (or as monetary value) and thus also represents energy.

In some circumstances another way of offsetting a specific uncertainty is made possible by providing corresponding information that removes the uncertainty. We may measure the information that is linked to a specific uncertainty by the work necessary to obtain this information (e.g. data analysis, education, research or detective work). In this sense information can also be expressed as work and therefore represents energy.

The following conservation principle extends the well-known principle of conservation of energy to the situation of markets including the notions of risk and information.

Fifth Principle. The value of assets, risk and information are different forms of energy, that is, the capacity to perform work, and they cannot be created or destroyed; although they can be transformed in other forms of energy.

This principle is indispensable for a global understanding of the relationships between economic and financial variables. Concepts such as work, kinetic and potential energy and power then appear naturally.

There is a need to relate different assets and markets, for example those of different countries via exchange rates. In the following relativity principle we postulate the important fact that all asset prices are relative.

Sixth Principle. If the above principles are valid in one market, they are equally valid in another market; then on the basis of market phenomena there is no way of determining absolute asset prices and only relative prices can be measured.

For instance, this principle allows one to consider systems of markets with different currencies that are linked via corresponding exchange rates. It also postulates a symmetry for asset prices, 
where the reciprocal of an asset price should also be an asset price. This can be easily seen in our example from relation (2.3), where the reciprocal of a price for a good is the price for the other good measured in units of the first good.

The principles mentioned here should be interpreted as philosophical statements. They can be used as guidelines for the construction of specific stochastic market models. More details will be given elsewhere.

\section{Acknowledgements}

The author wishes to thank David Heath and Chris Heyde for stimulating and encouraging discussions on the subject.

\section{References}

Black, F. AND Scholes, M. (1973). The pricing of options and corporate liabilities. J. Political Economy 81, $637-659$.

FÖLLMER, H. AND SCHWEIZER, M. (1991). Hedging of contingent claims under incomplete information. In Applied Stochastic Analysis, ed. M. Davis and R. Elliott (Stochastics Monographs 5). Gordon and Breach, London, pp. 389-414.

Föllmer, H. AND Sondermann, D. (1986). Hedging of non-redundant contingent claims. In Contributions to Mathematical Economics, ed. W. Hildenbrand and A. Mas-Colell. North Holland, Amsterdam, pp. $205-223$.

LINTNER, J. (1965). The valuation of risk assets and the selection of risky investments in stock portfolios and capital budgets. Rev. Econom. Statist. 47, 13-37.

Merton, R. (1973). An intertemporal capital asset pricing model. Economica 41, 867-888.

Platen, E. And Rebolledo, R. (1996). Principles for modelling financial markets. J. Appl. Prob. 33, $163-172$.

Ross, S. A. (1976). The arbitrage theory of capital asset pricing. J. Econom. Theory 13, 341-360.

Sharpe, W. F. (1964). Capital asset prices: a theory of market equilibrium under conditions of risk. J. Finance 19, $425-442$.

Yours sincerely,

ECKHARD PLATEN

Centre for Financial Mathematics, SMS, Australian National University, Canberra, ACT 0200, Australia. Email address: Eckhard.Platen@maths.anu.edu.au 